\title{
Optimization of Shortest Path Problem using Dijkstra's Algorithm in Imprecise Environment
}

\author{
Samir Dey \\ Department of Computer \\ Science and Engineering, \\ JIS University \\ Agarpara, Kolkata-700109, \\ West Bengal, India.
}

\author{
Sriza Malakar \\ Department of Computer \\ Science and Engineering, \\ Asansol Engineering College \\ Vivekananda Sarani, Asansol- \\ 713305, West Bengal, India.
}

\author{
Shibnath Rajak \\ Department of Computer \\ Science and Engineering, \\ Asansol Engineering College \\ Vivekananda Sarani, Asansol- \\ 713305, West Bengal, India.
}

\begin{abstract}
Dijkstra algorithm is a widely used algorithm to find the shortest path between two specified nodes in a network problem. In this paper, a generalized fuzzy Dijkstra algorithm is proposed to find the shortest path using a new parameterized defuzzification method. Here, we address most important issue like the decision maker's choice. A numerical example is used to illustrate the efficiency of the proposed algorithm.

Keywords: Dijkstra Algorithm; Generalized Trapezoidal Fuzzy Number; Shortest Path Problem.
\end{abstract}

\section{INTRODUCTION}

The shortest path problem (SPP) is the heart of a network's flow. In real life network, the main objective is to send the product with efficiently and with minimum budget between two predefined nodes. Dijkstra algorithm [9] is a widely used algorithm to find the shortest path between two specified nodes in a network problem. Crisp numbers are used to represent the costs traversing of edges to the network problem. Due to price fluctuation, bad weather and traffic problem, the information related to the edges of the network are vague in nature. To address this vagueness, Zadeh [1] was introduced the concept of fuzzy set. In last three decades, numerous research papers have been published in the domain of shortest path problem (SPP). Okada et al. [2] proposed possibility theory to solve the fuzzy SPP. Keshavarz et al. [3] demonstrated the techniques to convert generalized the fuzzy SPP in to a bi-level programming problem and its solution. A multiple constraint network problem was solved by Dou et al. [4]. Deng et al. [5] proposed Dijkstra algorithm using the ranked mean integration definition of fuzzy numbers to solve fuzzy SPPs. Furthermore, some works [6,7] based on heterogeneous forms of heuristic algorithm-based fuzzy arc values in network have been done. But decision makers choice was missing. Nayeem et al. [8] addressed decision makers choice in their work. In this paper, we have proposed parameterized fuzzy Dijkstra algorithm to solve fuzzy shortest path problem. In this fuzzy shortest path problem, the length of the edges is represented by generalized trapezoidal fuzzy number. A new defuzzification method is discussed based on total $\lambda$-integral value. Here decision maker can take a decision according their choice based on the value of the parameter $\lambda$.

\section{MATHEMATICAL PRELIMINARIES}

\subsection{Fuzzy Set}

Let $X$ denotes a universal set. Then the fuzzy subset $A$ in $X$ is a subset of order pairs $\AA^{\circ}=\left\{\left(x, \mu_{\alpha}(x)\right): x \in X\right\}$ where $\mu_{\mathcal{A}^{\circ}}: X \rightarrow[0,1]$ is called the membership function which assigns a real number $\mu_{\alpha(x)}(x)$ in the interval $[0,1]$ to each element $x \in X . A$ is non-fuzzy and $\mu_{A}(x)$ is identical to the characteristic function of crisp set. It is clear that the range of membership function is a subset of non-negative real numbers.

\subsection{Generalized Fuzzy Number (GFN)}

The generalized fuzzy number $\AA$ is a fuzzy subset of real line $R$, whose membership function $\mu_{\AA}(x)$ satisfies the following conditions:

(1) $\mu_{\AA}(x)$ is a continuous mapping from $R$ to the closed interval $[0,1]$.

(2) $\mu_{\AA}(x)=0$ where $-\infty<x \leq a$;

(3) $\mu_{\AA}(x)$ is strictly increasing with constant rate on $[a, b]$

(4) $\mu_{\AA}(x)=w$ where $b \leq x \leq c$

(5) $\mu_{\AA}(x)$ is strictly decreasing with constant rate on $[c, d]$;

(6) $\mu_{\AA}(x)=0$ where $d \leq x<\infty$.

Note: $\AA$ is a convex fuzzy set. It will be normalized for $w=1$.

If $\mathrm{w}=1$, the generalized fuzzy number $\AA$ is called a trapezoidal fuzzy number $(\mathrm{TrFN})$ denoted

$\AA=(a, b, c, d)$.

(i) If $a=b$ and $c=d$, then $\AA$ is called crisp interval $[a, b]$.

(ii) If $b=c$, then $\AA$ is called a generalized triangular fuzzy number $(\mathrm{GTFN})$ as $\stackrel{\AA}{\AA}=(a, b, c ; w)$

(iii) If $b=c, w=1$ then it is called a triangular fuzzy number $(\mathrm{TFN})$ as $\stackrel{\AA}{A}=(a, b, c)$. 
(iv) If $a=b=c=d$ and $w=1$, then $\AA$ is called a real number $a$.

\subsection{Generalized Trapezoidal Fuzzy Number (GFN)}

A $\operatorname{GTrFN} \AA \equiv(a, b, c, d ; w)$ is a fuzzy set of the real line $R$ whose membership function $\mu_{\AA}: R \rightarrow[0, w]$ is defined as

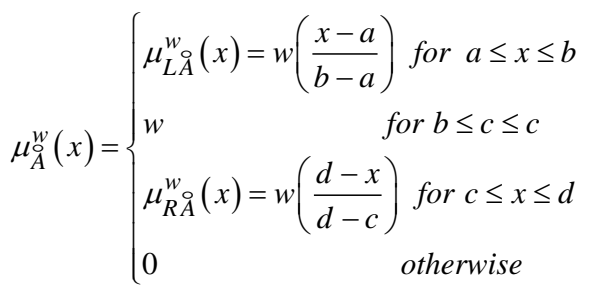

where $a<b<c<d$ and $w \in(0,1]$

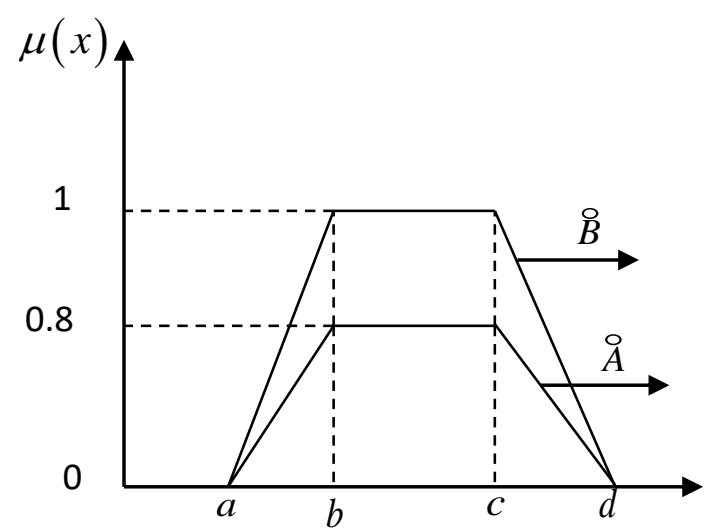

Figure 1: Two generalized trapezoidal fuzzy number $\stackrel{\AA}{A}$ and $\stackrel{\circ}{ }$

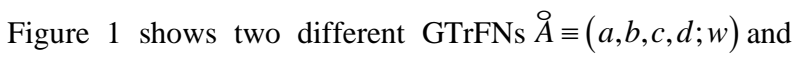
$\AA \equiv(a, b, c, d)$ which denote two different decision maker's opinions for different values of $w, 0<w \leq 1$. The values of $w$ represents the degree of confidence of the opinion of the decision maker.

\subsection{Defuzzification of Fuzzy Numbers with Respect to Their Total Integral Value}

Let $\lambda \in[0,1]$ be a pre-assigned parameter called the degree of optimism. The graded mean[10] value (or, total $\lambda$-integral value) of $\AA$ is defined as $I_{\lambda}(\AA)=\lambda I_{R}(\AA)+(1-\lambda) I_{L}(\AA)$ where $I_{R}(\AA)$ and $I_{L}(\AA)$ are the right and left interval values

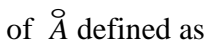

$$
\begin{aligned}
& I_{L}^{w}(\AA)=\left(\int_{0}^{w}\left(\mu_{L \AA}^{w}\right)^{-1} \alpha d \alpha\right) / w \\
& I_{R}^{w}(\AA)=\left(\int_{0}^{w}\left(\mu_{R \AA}^{w}\right)^{-1} \alpha d \alpha\right) / w
\end{aligned}
$$

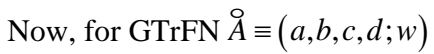

$\left(\mu_{L \AA}^{w}\right)^{-1} \alpha=a+\frac{\alpha}{w}(b-a)$ and $\left(\mu_{R \AA}^{w}\right)^{-1} \alpha=d-\frac{\alpha}{w}(d-c)$

Therefore, the left and right integral values are $I_{L}^{w}(\AA)=\left(\frac{a+b}{2}\right)$ and $I_{R}^{w}(\AA)=\left(\frac{c+d}{2}\right)$

Hence the total $\lambda$-integral value of $\AA$ is

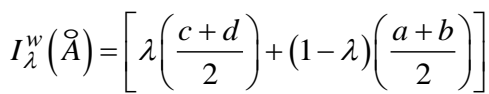

The total $\lambda$-integral value is a convex combination of the right and left integral values through the degree of optimism. The left integral value is used to reflect the pessimistic viewpoint and the right integral value is used to reflect the optimistic viewpoint of the decision-maker. A large value of $\lambda$ specifies the higher degree of optimism. For instance, when $\lambda=1$, the total integral value

$I_{1}^{w}(\AA)=\left(\frac{c+d}{2}\right)=I_{R}^{w}(\AA)$ represents an optimistic viewpoint.

On the other hand, when $\lambda=0$, the total $\lambda$-integral value is $I_{0}^{w}(\AA)=\left(\frac{a+b}{2}\right)=I_{L}^{w}(\AA) \quad$ represents a pessimistic viewpoint. When $\lambda=0.5$ the total $\lambda$-integral is $I_{0.5}^{w}(\AA)=\left(\frac{a+b+c+d}{4}\right)$. It reflects a moderately optimistic decision-makers viewpoint and is the same as the defuzzification of the fuzzy number $\stackrel{\AA}{\text {. }}$

\section{PROPERTIES}

\section{Property: 3.1}

(a) If $\operatorname{GTrFN} \stackrel{O}{U}=\left(u_{1}, u_{2}, u_{3}, u_{4} ; w\right)$ and $y=k u$ (with $k<0$ ) then $y^{\mathscr{O}} k u^{\prime}$ is $\operatorname{aTrFN}\left(k u_{1}, k u_{2}, k u_{3}, k u_{4} ; w\right)$.

(b) If $y=k u($ with $k<0)$ then $\mathscr{O}^{\circ} k u^{\prime}$ is a $\operatorname{GTrFN}\left(k u_{1}, k u_{2}, k u_{3}, k u_{4} ; w\right)$.

\section{Proof:}

(a) When $k>0$ with the transformation $y=k u$, we can find the membership function of fuzzy set $y^{0} k u^{\prime}$ by $\alpha$-cut method.

$\alpha$-cut of $U^{\circ}$ is

$\left[\left(\mu_{L U}^{w}\right)^{-1} \alpha,\left(\mu_{R U}^{w}\right)^{-1} \alpha\right]=\left[u_{1}+\frac{\alpha}{w}\left(u_{2}-u_{1}\right), u_{4}-\frac{\alpha}{w}\left(u_{4}-u_{3}\right)\right]$ for any $\alpha \in[0,1]$

i.e. $u \in\left[u_{1}+\frac{\alpha}{w}\left(u_{2}-u_{1}\right), u_{4}-\frac{\alpha}{w}\left(u_{4}-u_{3}\right)\right]$

So $y(=k u) \in\left[k u_{1}+\frac{\alpha}{w}\left(k u_{2}-k u_{1}\right), k u_{4}-\frac{\alpha}{w}\left(k u_{4}-k u_{3}\right)\right]$.

Thus, we get the membership function of $y^{O}=k u^{\prime}$ as 


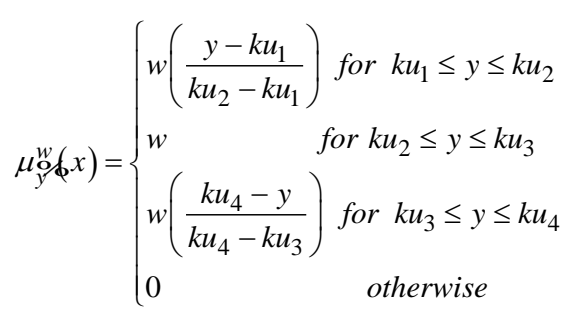

(b) Similarly, we can prove that $y=k u, k<0$ then

$$
\mu_{y}(x)= \begin{cases}w\left(\frac{y-k u_{1}}{k u_{2}-k u_{1}}\right) & \text { for } k u_{2} \leq y \leq k u_{1} \\ w & \text { for } k u_{3} \leq y \leq k u_{2} \\ w\left(\frac{k u_{4}-y}{k u_{4}-k u_{3}}\right) & \text { for } k u_{4} \leq y \leq k u_{3} \\ 0 & \text { otherwise }\end{cases}
$$

\section{Property 3.2}

If $\stackrel{\AA}{1}_{1}=\left(a_{1}, b_{1}, c_{1}, d_{1} ; w_{1}\right)$ and $\AA_{2}=\left(a_{2}, b_{2}, c_{2}, d_{2} ; w_{2}\right)$ then $\AA_{1} \oplus \AA_{2}$ is a fuzzy number $\left(a_{1}+a_{2}, b_{1}+b_{2}, c_{1}+c_{2}, d_{1}+d_{2} ; \min \left(w_{1}, w_{2}\right)\right)$.

\section{Proof:}

With the transformation $y=x_{1}+x_{2}$ we can find the membership function of fuzzy set $\mathscr{Y}^{\circ} \AA_{1} \oplus \AA_{2}$ by $\alpha$-cut method.

$\alpha$-cut of $\AA_{1}$ is

$\left[\left(\mu_{L \AA_{1}}^{w_{1}}\right)^{-1} \alpha,\left(\mu_{R \AA_{1}}^{w_{1}}\right)^{-1} \alpha\right]=\left[a_{1}+\frac{\alpha}{w_{1}}\left(b_{1}-a_{1}\right), d_{1}-\frac{\alpha}{w_{1}}\left(d_{1}-c_{1}\right)\right]$

$\forall \alpha \in[0,1]$

i.e. $x_{1} \in\left[a_{1}+\frac{\alpha}{w_{1}}\left(b_{1}-a_{1}\right), d_{1}-\frac{\alpha}{w_{1}}\left(d_{1}-c_{1}\right)\right]$.

$\alpha$-cut of $\stackrel{\circ}{2}_{2}$ is

$\left[\left(\mu_{L \AA_{2}}^{w_{1}}\right)^{-1} \alpha,\left(\mu_{R \AA_{2}}^{w_{1}}\right)^{-1} \alpha\right]$

$=\left[a_{2}+\frac{\alpha}{w_{2}}\left(b_{2}-a_{2}\right), d_{2}-\frac{\alpha}{w_{2}}\left(d_{2}-c_{2}\right)\right]^{\forall \alpha \in[0,1]}$

i.e. $x_{2} \in\left[a_{2}+\frac{\alpha}{w_{2}}\left(b_{2}-a_{2}\right), d_{2}-\frac{\alpha}{w_{2}}\left(d_{2}-c_{2}\right)\right]$.

So, $y\left(=x_{1}+x_{2}\right) \in\left[\begin{array}{l}a_{1}+a_{2}+\frac{\alpha}{w}\left(\left(b_{1}-a_{1}\right)+\left(b_{2}-a_{2}\right)\right), \\ d_{1}+d_{2}-\frac{\alpha}{w}\left(\left(d_{1}-c_{1}\right)+\left(d_{2}-c_{2}\right)\right)\end{array}\right]$ where $w=\min \left(w_{1}, w_{2}\right)$. Therefore, we have

$$
\begin{aligned}
& \alpha=w\left(\frac{y-a_{1}-a_{2}}{b_{1}+b_{2}-a_{1}-a_{2}}\right), a_{1}+a_{2} \leq y \leq b_{1}+b_{2}, \text { and } \\
& \alpha=w\left(\frac{d_{1}+d_{2}-y}{d_{1}+d_{2}-c_{1}-c_{2}}\right), c_{1}+c_{2} \leq y \leq d_{1}+d_{2}
\end{aligned}
$$

So, we have the membership function of $\mathscr{Y}^{\circ} \AA_{1} \oplus \AA_{2}$ is

$$
\mu_{\mathrm{o} g}(x)= \begin{cases}w\left(\frac{y-a_{1}-a_{2}}{b_{1}+b_{2}-a_{1}-a_{2}}\right) & \text { for } a_{1}+a_{2} \leq y \leq b_{1}+b_{2} \\ w & \text { for } b_{1}+b_{2} \leq y \leq c_{1}+c_{2} \\ w\left(\frac{d_{1}+d_{2}-y}{d_{1}+d_{2}-c_{1}-c_{2}}\right) & \text { for } c_{1}+c_{2} \leq y \leq d_{1}+d_{2} \\ 0 \quad & \text { otherwise }\end{cases}
$$

Thus we have

$\AA_{1} \oplus A_{2}=\left(a_{1}+a_{2}, b_{1}+b_{2}, c_{1}+c_{2}, d_{1}+d_{2} ; \min \left(w_{1}, w_{2}\right)\right)$. Note: If we have the transformation $\mathscr{O}^{\circ} k_{1} \AA_{1} \oplus k_{2} A_{2} \quad\left(k_{1}, k_{2}\right.$ are (not all zero) real numbers) then the fuzzy set $y=\mathbb{O}_{1} \AA_{1} \oplus k_{2} A_{2}$ is the following GTrFN:

(i) $k_{1}>0, k_{2} \geq 0$ or $k_{1} \geq 0, k_{2}>0$

$$
y=\left(k_{1} a_{1}+k_{2} a_{2}, k_{1} b_{1}+k_{2} b_{2}, k_{1} c_{1}+k_{2} c_{2}, k_{1} d_{1}+k_{2} d_{2}: w\right)
$$

(ii) $k_{1}>0, k_{2} \leq 0$ or $k_{1} \geq 0, k_{2}<0$,

$$
\mathbb{Y}^{-}\left(k_{1} a_{1}+k_{2} d_{2}, k_{1} b_{1}+k_{2} c_{2}, k_{1} c_{1}+k_{2} b_{2}, k_{1} d_{1}+k_{2} a_{2}: w\right)
$$

(iii) $k_{1}<0, k_{2} \geq 0$ or $k_{1} \leq 0, k_{2}>0$,

$$
y=\left(k_{1} d_{1}+k_{2} d_{2}, k_{1} c_{1}+k_{2} c_{2}, k_{1} b_{1}+k_{2} b_{2}, k_{1} a_{1}+k_{2} a_{2}: w\right)
$$

\section{DIJKSTRA'S ALGORITHM}

Let $G=(V, E)$ be a connected graph. Let $a$ and $b$ be any two vertices of $G$ where ' $a$ ' is the starting point and $b$ be the ending point. Let $L(x)$ denote the label of the vertex ' $x$ ' which represents the length of the shortest path form the vertex ' $a$ ' to ' $x$ ' denote the weight of the edge $e_{i j}=\left(v_{i}, v_{j}\right)$

Step 1: Let $P=\phi$, where $P$ is the set of vertices having permanent labels and $T=\{$ all vertices of the graph $G\}$. Initially set the permanent label to a,i.e., $L(a)=0, L(x)=\infty \forall x \in T$ and $x \neq a$.

Step 2: Select the vertex $v$ in $T$ which has the smallest label, which is called the permanent label of $v(i . e ., u(v))$; then $P=P U(v)$ and $T=T-\{v\}$

Then find the smallest label in $T$.The new label of vertex $x$ in $T$ is

$$
L(x)=\min \{\text { old } L(x), L(v)+w(v, x)\}
$$


International Journal of Computer Applications Technology and Research

Volume 10- Issue 10, 216-221, 2021, ISSN: - 2319-8656

\section{APPLICATION TO AIRPORT'S CARGO GROUND OPERATION SYSTEM}

As the volume of cargo traffic has grown and the demand for cargo transport continues to rise, surface congestion has become an increasing problem, within an airport's cargo terminal. If an airport terminal's internal operations and service systems are inefficient, there will be a delay in ground operations. Therefore, cargo operations time needs to be shortened and passengers' luggage must be processed before cargo goods in order to maintain customer satisfaction. Fig.2.shows an international airport cargo terminal's ground operation procedures network. With the set of nodes $\mathrm{N}=\{\mathrm{A}, \mathrm{B}, \mathrm{C}, \mathrm{D}, \mathrm{E}, \mathrm{F}\}$, the fuzzy activity time for each activity is shown in table 1 .

Table 1: Fuzzy activity time for each activity

\begin{tabular}{|c|c|c|}
\hline Activity & Fuzzy activity time & $\lambda$ - Integral Value \\
\hline$A-B$ & $(1,3,3,7 ; 0.8)$ & $4 \lambda+(1-\lambda) 1.6$ \\
\hline$A-C$ & $(2,3,3,4 ; 0.6)$ & $2.1 \lambda+(1-\lambda) 1.5$ \\
\hline$B-C$ & $(3,5,6,8 ; 0.8)$ & $5.6 \lambda+(1-\lambda) 3.2$ \\
\hline$B-E$ & $(2,4,5,6 ; 0.8)$ & $4.4 \lambda+(1-\lambda) 1.6$ \\
\hline$C-D$ & $(1,3,3,4 ; 0.8)$ & $2.8 \lambda+(1-\lambda) 1.6$ \\
\hline$B-D$ & $(1,2,3,6 ; 0.6)$ & $2.7 \lambda+(1-\lambda) 0.9$ \\
\hline$D-E$ & $(1,3,4,5 ; 0.6)$ & $2.7 \lambda+(1-\lambda) 1.2$ \\
\hline$D-F$ & $(1,2,2,5 ; 0.8)$ & $2.8 \lambda+(1-\lambda) 1.2$ \\
\hline$E-F$ & $(4,5,5,8 ; 0.8)$ & $5.2 \lambda+(1-\lambda) 3.6$ \\
\hline
\end{tabular}

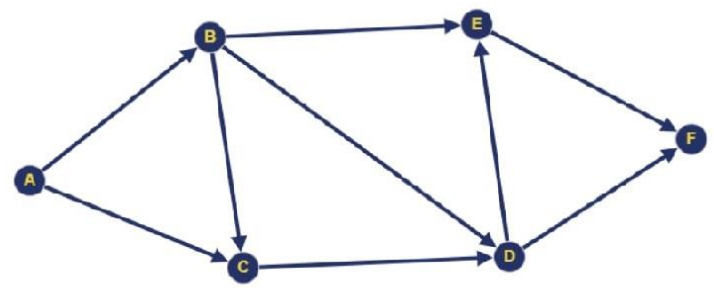

Fig-2. Airport cargo terminal's ground operation network

\begin{tabular}{|l|l|l|l|l|l|}
\hline \multirow{2}{*}{\begin{tabular}{l} 
Activit \\
\cline { 2 - 6 }
\end{tabular}} & \multicolumn{5}{|c|}{$\lambda-$ Integral Value } \\
\cline { 2 - 6 } & $\lambda=0$ & $\lambda=0.25$ & $\lambda=0.5$ & $\lambda=0.75$ & $\lambda=1.0$ \\
\hline$A-B$ & 1.6 & 2.2 & 2.8 & 3.4 & 4 \\
\hline$A-C$ & 1.5 & 1.65 & 1.8 & 1.95 & 2.1 \\
\hline$B-C$ & 3.2 & 3.8 & 4.4 & 5 & 5.6 \\
\hline$B-E$ & 1.6 & 2.3 & 3 & 3.7 & 4.4 \\
\hline$C-D$ & 1.6 & 1.9 & 2.2 & 2.5 & 2.8 \\
\hline$B-D$ & 0.9 & 1.35 & 1.8 & 2.25 & 2.7 \\
\hline$D-E$ & 1.2 & 1.575 & 1.95 & 2.325 & 2.7 \\
\hline$D-F$ & 1.2 & 1.6 & 2 & 2.4 & 2.8 \\
\hline$E-F$ & 3.6 & 4 & 4.4 & 4.8 & 5.2 \\
\hline
\end{tabular}

Solution: Now using Dijkstra's algorithm to find the shortest path from vertex $A$ to vertex $F$ for $\lambda=0$ integral value.
Step 1: $P=\varphi$ and $T=\{A, B, C, D, E, F\}$

Let the label of $A$,i.e, $L(A)=0$ and $L(x)=\infty \forall x \in T(x \neq A)$

Step 2: $V=A$, the permanent label is $L(A)=0$

$\therefore \quad P=\{A\}$ and $T=\{B, C, D, E, F\}$

$L(B)=$

$\min \{\operatorname{old} L(B), L(A)+w(A, B)\}=\min \{\infty, 0+1.6\}=1.6$

. $L(C)=$

$\therefore \min \{$ old $L(C), L(A)+w(A, C)\}=\min \{\infty, 0+1.5\}=1.5$

$L(D)=$

$\min \{$ old $L(D), L(A)+w(A, D)\}=\min \{\infty, 0+\infty\}=\infty$

Similarly, $L(E)=L(F)=\infty$

We see that the minimum label is $L(C)=1.5$

Step 3: $V=C$, the permanent label is $L(C)=1.5$

$\therefore \quad P=\{A, C\}$ and $T=\{B, D, E, F\}$

$\therefore$

$L(B)=$

$\min \{$ old $L(B), L(C)+w(C, B)\}=\min \{1.6,1.5+\infty\}=1.6$

$L(D)=$

$\min \{$ old $L(D), L(C)+w(C, D)\}=\min \{\infty, 1.5+1.6\}=3.1$

$L(E)=$

$\min \{$ old $L(E), L(C)+w(C, E)\}=\min \{\infty, 1.5+\infty\}=\infty$

$L(F)=$

$\min \{\operatorname{old} L(F), L(C)+w(C, F)\}=\min \{\infty, 1.5+\infty\}=\infty$

We see that minimum label is $L(B)=1.6$.

Step 4: $V=B$, the permanent label is $L(B)=1.6$

$\therefore \quad P=\{A, B, C\}$ and $T=\{D, E, F\}$

$\therefore$

$L(D)=$

$\min \{\operatorname{old} L(D), L(B)+w(B, D)\}=\min \{3.1,1.6+0.9\}=2.5$

$L(E)=$

$\min \{$ old $L(E), L(B)+w(B, E)\}=\min \{\infty, 1.6+1.6\}=3.2$

$L(F)=$

$\min \{$ old $L(F), L(B)+w(B, F)\}=\min \{\infty, 1.6+\infty\}=\infty$

Hence, the minimum label is $L(D)=2.5$.

Step 5: $V=D$, the permanent label is $L(D)=2.5$

$\therefore \quad P=\{A, B, C, D\}$ and $T=\{E, F\}$. 
$\therefore$

$L(E)=$

$\min \{$ old $L(E), L(D)+w(D, E)\}=\min \{3.2,2.5+1.2\}=3.2$

$L(F)=$

$\min \{$ old $L(F), L(D)+w(D, F)\}=\min \{\infty, 2.5+1.2\}=3.7$

Hence, the minimum label is $L(E)=3.2$

Step 6: $V=E$ the permanent label is $L(E)=3.2$

$$
\begin{array}{ll}
\therefore & P=\{A, B, C, D, E\} \text { and } T=\{F\} \\
\therefore & L(F)=\min \{\text { old } L(F), L(E)+w(E, F)\} \\
& =\min \{3.7,3.2+3.6\}=3.7
\end{array}
$$

Hence, the permanent label of $F$ is 3.7 which is the shortest path distance from the vertex ' $A$ ' to ' $F$ '

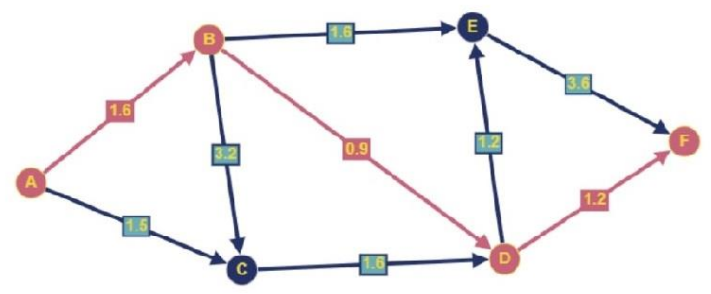

Fig-3. Optimum shortest path of Airport cargo terminal's ground operation network for Integral Value $\lambda=0$

Using Dijkstra's algorithm, the optimum solution are as follows for different $\lambda$-integral value

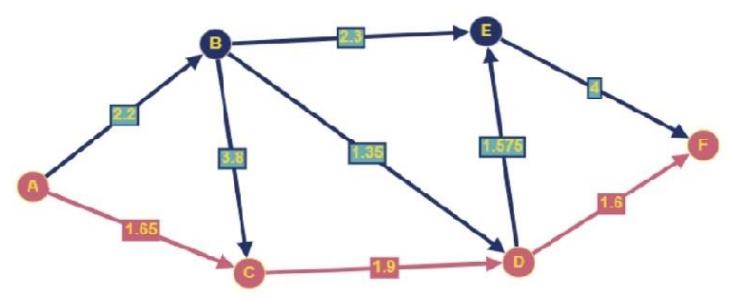

Fig-4. Optimum shortest path of Airport cargo terminal's ground operation network for Integral Value $\lambda=0.25$

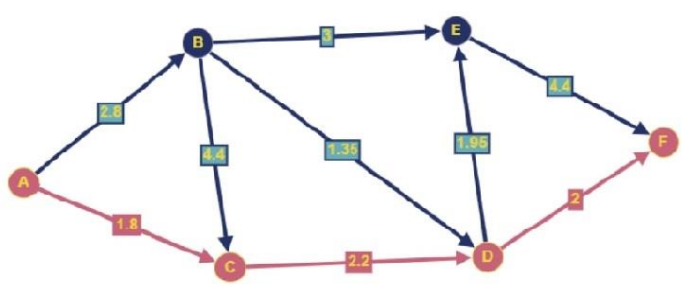

Fig-5. Optimum shortest path of Airport cargo terminal's ground operation network for Integral Value $\lambda=0.5$

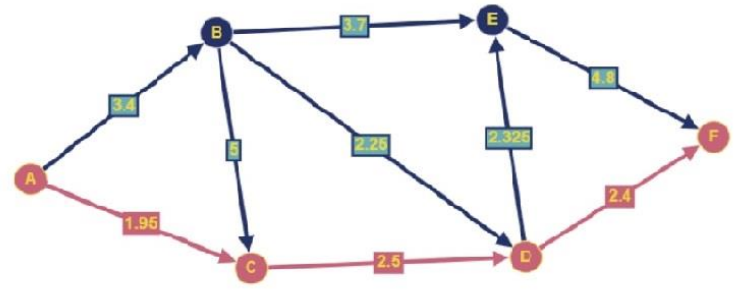

Fig-6. Optimum shortest path of Airport cargo terminal's ground operation network for Integral Value $\lambda=0.75$

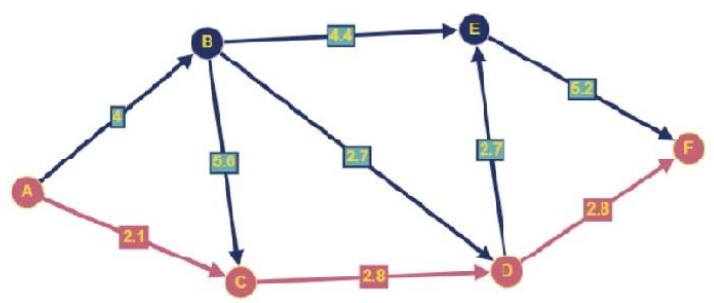

Fig-7. Optimum shortest path of Airport cargo terminal's ground operation network for Integral Value $\lambda=1.0$

The optimal solution of the fuzzy project network model for different values of $\lambda$ are presented in table 2 .

Table 2. optimal solution of the fuzzy Airport cargo terminal's ground operation network

\begin{tabular}{|c|c|c|c|}
\hline Test & Critical Path & Duration & $\begin{array}{c}\text { Model } \\
\text { Figure }\end{array}$ \\
\hline $\begin{array}{c}\text { Optimistic i.e } \\
\lambda=1\end{array}$ & A-C-D-F & 7.7 & Fig-7 \\
\hline $\begin{array}{c}\text { About } \\
\text { optimistic i.e } \\
\lambda=0.75\end{array}$ & A-C-D-F & 6.85 & Fig-6 \\
\hline $\begin{array}{c}\text { Moderate i.e } \\
\lambda=0.5\end{array}$ & A-C-D-F & 6 & Fig-5 \\
\hline $\begin{array}{c}\text { About } \\
\text { pessimistic i.e } \\
\lambda=0.25\end{array}$ & A-C-D-F & 5.15 & Fig-4 \\
\hline $\begin{array}{c}\text { Pessimistic i.e } \\
\lambda=0\end{array}$ & A-B-D-F & 3.7 & Fig-3 \\
\hline
\end{tabular}

\section{CONCLUSION}

This paper presents a simple approach to solve a Cargo network problem with fuzzy number (generalized trapezoidal fuzzy number) activity times that are more realistic than crisp ones. On the basis of $\lambda$-integral value, the fuzzy Cargo network problem is transformed into a crisp Cargo network problem. We than solved the cargo problem by Dijkstra's algorithm. Here decision maker may obtain the optimal project duration according to his/her expectations of optimistic/pessimistic/moderate

\section{ACKNOWLEDGMENTS}

Our thanks to authority of JIS University and Asansol Engineering College for their support.

\section{REFERENCES}

[1] Zadeh, L.A. Fuzzy sets, Information and Control 1965, 8, 338-353. 
[2] Okada, S.; Soper, T. A shortest path problem on a network with fuzzy arc lengths. Fuzzy Sets Syst. 2000, 109, 129-140.

[3] Keshavarz, E.; Khorram, E. A fuzzy shortest path with the highest reliability. J. Comput. Appl. Math. 2009, 230, 204-212.

[4] Dou, Y.; Zhu, L.; Wang, H.S. Solving the fuzzy shortest path problem using multi-criteria decision method based on vague similarity measure. Appl. Soft Comput. 2012, $12,1621-1631$.

[5] Deng, Y.; Chen, Y.; Zhang, Y.; Mahadevan, S. Fuzzy Dijkstra algorithm for shortest path problem under uncertain environment. Appl. Soft Comput. 2012, 12, 1231-1237.

[6] Ebrahimnejad, A.; Karimnejad, Z.; Alrezaamiri, H. Particle swarm optimisation algorithm for solving shortest path problems with mixed fuzzy arc weights. Int. J. Appl. Decis. Sci. 2015, 8, 203-222.

[7] Ebrahimnejad, A.; Tavana, M.; Alrezaamiri, H. A novel artificial bee colony algorithm for shortest path problems with fuzzy arc weights. Measurement 2016, 93, 48-56.

[8] Nayeem, S.M.A., Pal,M., shortest path problem on a network with imprecise edge weight, Fuzzy Optimization Decision Making 2005, 4, 293-312.

[9] Dijkstra, E.W. A note on two problems in connexion with graphs. Numer. Math. 1959, 1, 269-271.

[10] Hsieh, C.H.; Chen, S.H. Similarity of Generalized Fuzzy Numbers with Graded Mean Integration Representation. Proceedings of the 8th International Fuzzy Systems Association World Congress, Taipei 1999, 551-555. 